URL www.una.ac.cr/mhsalud

\title{
SOMATOTIPO EN PIRAGÜISTAS ÉLITE DE CUBA
}

\author{
Arián Ramón Aladro Gonzalvo ${ }^{1}$, Miriam Machado Díaz ${ }^{2}$ y Edgar Bueno Fernández ${ }^{3}$ \\ ${ }^{1}$ Licenciado Rehabilitador y Entrenador Deportivo. Centro Provincial de Medicina Deportiva de \\ Cienfuegos.Cuba,pgonzalvo@ucf.edu.cu, aladro80@fastmail.es \\ ${ }^{2}$ Doctora. Especialista en Medicina Física y Rehabilitación. Centro Provincial de Medicina Deportiva de \\ Cienfuegos. Cuba \\ ${ }^{3}$ Doctor en Ciencias de la Cultura Física. Universidad de Cienfuegos. Cuba
}

\section{RESUMEN}

\begin{abstract}
Algunas características físicas están ligadas al máximo desempeño deportivo, dando fundamento a un llamado prototipo morfológico y su conocimiento resulta de suma importancia a los profesionales de la actividad física. Por tal motivo, la intención del presente trabajo fue evidenciar si los somatotipos de los atletas élite de canotaje de Cuba, se relacionan con referencias internacionales, tales como estudios realizados en España (Esparza, 1993), Canadá (Carter, 1999), Australia (Ackland y otros, 2000), Argentina (Lentini y otros, 2002) y EE.UU. (Vaccaro y otros, 2000). Fueron evaluados 30 deportistas los cuales se distribuían en 9 féminas de kayak, y 21 masculinos de canoa y kayak.

Dicho estudio condujo a reconocer la gran heterogeneidad existente en esta población, a juzgar por el comportamiento de los somatotipos a partir de comparaciones intragrupales. Al comparar con otros estudios de referencia el componente endomórfico proporcionó la diferencia. Esto permitió concluir que existe necesidad de ejecutar acciones que modifiquen dicho componente, para así lograr un acercamiento de los atletas cubanos al perfil antropométrico que exhiben equipos de élite mundial.
\end{abstract}

PALABRAS CLAVES: Somatotipo piragüistas, piragüistas élite, Somatotipo kayak-canoa, Somatotipo cubano.

\section{SOMATOTYPE OF ELITE CUBAN PADDLERS}

\begin{abstract}
Some physical characteristics are related to maximum sport performance, which has resulted in what has been denominated the morphological prototype. Knowing about this concept is of great relevance for physical activity professionals. Consequently, the purpose of this research was to give evidence as to whether the somatotypes of elite Cuban paddlers are related to international studies done in Spain (Esparza, 1993), Canada (Carter, 1999), Australia (Ackland et al, 2000), Argentina (Lentini et al, 2002), and the United States (Vaccaro et al, 2000). A total of 30 athletes were evaluated including 9 female kayakers and 21 male canoeists and kayakers.

This study shows the significant heterogeneity found in this population based on the behavior of the somatotypes when contrasting the groups. Compared to the other reference studies, the endomorphic component proved to be the differentiating factor in this research. The foregoing allowed us to conclude that actions should be taken to modify this component in a way that Cuban athletes are closer to the anthropometric profile exhibited by international elite teams.
\end{abstract}

KEY WORDS: Paddler somatotype, elite paddlers, kayaking-canoeing somatotype, Cuban somatotype.

\section{INTRODUCCIÓN}

La antropometría surge como herramienta para cuantificar las formas corporales y dimensiones, convirtiéndose en un método valioso en el área de las mediciones corporales de los deportistas o los que no lo son. Se basa en la toma con instrumental acorde, de perímetros musculares, pliegues cutáneos, diámetros óseos, longitudes de los miembros, peso corporal y talla (Lentini, Gris, Cardey, Aquilino, Dolce, 2006; Parajón, 2002).Esta disciplina de la antropología como afirman Berral (2005a) Parajón (2002) es la ciencia que describe la estructura morfológica del individuo en su desarrollo longitudinal y las modificaciones provocadas por el entrenamiento. Es considerada una disciplina básica para la solución de los problemas relacionados con el crecimiento, el desarrollo, el ejercicio, la nutrición y la performance. 


\section{MHSalud}

URL www.una.ac.cr/mhsalud

El estudio antropológico de los deportistas parte de la premisa de que la actividad física deportiva es un factor ambiental que modifica las características de la persona que lo practica. Autores como Berral (2005a) y García y Rodríguez (2004) sostienen que cada disciplina deportiva requiere de ciertas estructuras, proporcionalidad y silueta para lograr un buen desempeño y por esta razón expresan que en la medida en que se conozcan las particularidades del desarrollo de cada atleta, se contribuye al conocimiento de sus posibilidades para ejecutar esfuerzos físicos.

Dentro de ese análisis aparece la biotipología como pilar indiscutido, ofreciendo a través del método de HeathCarter la posibilidad de catalogar a los individuos por sus tres elementos esenciales, la Endomorfia (tendencia a la obesidad), la Mesomorfia componente (tendencia al desarrollo músculo-esquelético relativo) y la Ectomorfia (tendencia a la linealidad relativa). El somatotipo o biotipo es un sistema para valorar la morfología del cuerpo que permite distinguir fácilmente la figura exterior del individuo. Según los valores encontrados y dependiendo del predominio de uno u otro componente, Bárbara Heath y J.E.L Carter presentaron en 1967 trece categorías diferentes de somatotipo (Martínez, 1987).

En un sentido, el prototipo morfológico es una estructura corporal que se adapta de la mejor manera ante las exigencias de un deporte. Es el soporte indispensable para la obtención de los mayores logros desde el punto de vista del rendimiento físico. Por consiguiente un gran desafío para los cineantropometristas y los estudiosos de las ciencias deportivas, es la comprensión de los rasgos somáticos que diferencien aspectos relevantes, para poder establecer la asociación entre una dimensión corporal y el mejor desempeño dinámico. En el canotaje el análisis del somatotipo brinda razones coherentes para el estudio del rendimiento físico desde que el atleta inicia su actividad de entrenamiento sistemático para su participación en regatas nacionales y con mayor profundidad para las internacionales; controlando los cambios y evaluando las restricciones estructurales que podrían afectar la actuación, o tener relación con la salud o el bienestar físico.

Un estudio objetivo y real sobre la participación y los resultados obtenidos a partir de los Juegos Olímpicos de Moscú 1980 y otras competencias internacionales de gran alcance, hacen significativo que, actualmente pocos palistas cubanos puedan corresponderse al nivel de los mejores atletas a nivel internacional (Matveev, $2000 \mathrm{y}$ Romero, 2001).

Pese a los resultados obtenidos en este deporte a nivel nacional e internacional, no hay una base de datos importante desde el punto de vista antropométrico que sirva de guía a los entrenadores de la Federación de Canotaje de Cuba, y unido a esto existe escasa información en la literatura científica relacionada con las características biotipológicas, la composición corporal y proporcionalidad de los palistas (kayak / canoa) de alto nivel competitivo. Al mismo tiempo sería interesante comprobar si existe relación entre la distribución de los componentes del somatotipo y el resultado deportivo, ya que no se encontraron estudios que declararan la correlación de dichos componentes con otras variables predictoras. Incluir este último factor en el componente analítico del estudio, reduciría la emisión de criterios subjetivos; no obstante estos datos son esenciales para el desarrollo de los atletas en su preparación para la competición, o para utilizarlos con el desarrollo del talento. Si se empleara el método científico de determinación del somatotipo como evaluación dentro del programa anual de entrenamientos, daría una clara visión de cómo orientar la preparación de forma individualizada para cada atleta, e influir en el rendimiento deportivo de los mismos.

Tales consideraciones, se convierten en el foco de atención del presente estudio, en el que se pretende determinar el somatotipo de los piragüistas de élite de Cuba, basado en una descripción estadística que permita exponer, aquellas características biotipológicas que constituyan fuente de perfeccionamiento de los programas de preparación.

\section{METODOLOGÍA}




\section{MHSalud}

URL www.una.ac.cr/mhsalud

Sujetos: se seleccionaron como muestra a los 30 atletas que conforman la preselección nacional de canotaje en Cuba, con sede en el Centro Nacional de Entrenamiento de Alto Rendimiento de Remo y Canotaje en provincia Habana. De ellos pertenecen a la modalidad de kayak 9 mujeres y 11 hombres, y a la canoa 10 masculinos. La investigación tuvo lugar en la etapa de preparación general, en noviembre del 2006.

Instrumentos: para recolectar la información se confeccionó una ficha antropométrica (Anexo 1), donde se recogieron datos generales como: edad, sexo, modalidad, longevidad deportiva, etc.; y datos específicos: peso, talla, pliegues, perímetros y diámetros. Además se adjuntó a la ficha antropométrica un texto de autorización, que el sujeto firmaría, para poder utilizar los datos con fines de investigación.

Como criterio de resultados deportivos relevantes se tomó a medallistas en Juegos Panamericanos y Campeonatos Pre-olímpicos; medallista o finalista en Copas del Mundo y Campeonatos Mundiales; y medallista o finalista en Juegos Olímpicos (Ver Anexo 2).

Se efectuó la valoración antropométrica siguiendo las instrucciones establecidas por el Manual Oficial de Cineantropometría (International Standards for Anthropometric Assessment Unerdale, 2001) y de la Federación Española de Medicina Deportiva [FEMEDE] (Esparza, 1993). Todas las mediciones fueron tomadas unilateralmente del lado derecho, según las escuelas canadiense y estadounidense, por un único observador y un recolector de datos.

La variable peso se midió con una báscula (SECA. Alemania) con precisión de fracciones de $100 \mathrm{~g}$ para el peso y peso máximo de 150 kilogramos. La talla se midió con un tallímetro (SECA. Alemania) con precisión de fracciones de $0.1 \mathrm{~cm}$ para la talla. Para precisar los puntos anatómicos y otras marcas de referencia se utilizó un lápiz demográfico.

Los pliegues (tríceps, subescapular, suprailíaco y medial de la pierna) se midieron, por duplicado, con un lipómetro Harpenden Skinfold Caliper con amplitud de 0 a $40 \mathrm{~mm}$, graduación de $0.2 \mathrm{~mm}$. Los perímetros (brazo contraído y pierna) también se midieron por duplicado, con una cinta métrica flexible England. Los diámetros (biepicondileo de húmero y bicondileo de fémur) se determinaron con un paquímetro (Holtain Ltd. Reino Unido) de corredera, graduado de profundidad en sus ramas de $50 \mathrm{~mm}$, con capacidad de medida de 0 a $259 \mathrm{~mm}$.

Procedimientos: se utilizó el método de Heath y Carter que surge a partir de una modificación que Bárbara Heath (1948-1953) hizo del método fotoscópico de Sheldon. Este es el más utilizado desde entonces por ser el más rápido, práctico y económico, y como ejemplo están los numerosos estudios en distintos Juegos Olímpicos, como en México-68 (Carter, 1990), Montreal-76 (Carter, 1984) y Sydney-02 (Ackaland y colaboradores, 2001).

Análisis estadístico: Análisis del somatotipo: una vez calculados los valores de los tres componentes del somatotipo de los evaluados/as, se hace un análisis de los mismos, a través de procedimientos o estudios estadísticos, en el orden individual o por grupos (Berral 2005a Esparza, 1993; Gris, 2001). El procesamiento estadístico consistió en un análisis descriptivo, donde se determinaron medias, porcientos, desviación estándar, índice de dispersión y distancias de dispersión. Además se correlacionó el resultado deportivo con el somatotipo de toda la población, mediante el coeficiente de correlación de Spearman con un valor de $\mathrm{p} \leq 0.05$. Para ello se utilizó el paquete informático SPSS 11.0 para Windows®.

Análisis individual del somatotipo: Distancia de dispersión del somatotipo. Compara el somatotipo individual con el somatotipo medio. $\mathrm{SDD}=\sqrt{ }\left(3\left(\mathrm{X}_{1}-\mathrm{X}_{2}\right)^{2}+\left(\mathrm{Y}_{1}-\mathrm{Y}_{2}\right)^{2}\right)$.

Análisis en grupo del somatotipo: Somatotipo medio. Corresponde a la media de cada uno de los componentes de los individuos del grupo. 


\section{MHSalud}

URL www.una.ac.cr/mhsalud

Índice de Dispersión del somatotipo (SDI). Corresponde a la media de las Distancias de Dispersión del somatotipo (SDD) de los individuos del grupo estudiado respecto a su somatotipo medio. SDI $=\sum \mathrm{SDD} / \mathrm{n}$. Si el valor del SDI $\geq 2$ se puede entender que la muestra no es homogénea y que existen diferencias significativas entre los individuos que la integran.

Distancia de dispersión del somatotipo medio. En este caso se comparan las coordenadas X y Y del somatotipo

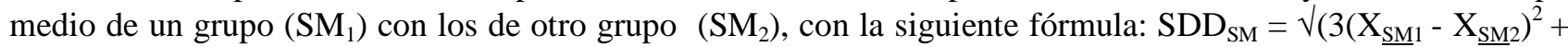
$\left.\left(\mathrm{Y}_{\underline{\mathrm{SM} 1}}-\mathrm{Y}_{\underline{\mathrm{SM}} 2}\right)^{2}\right)$. Si $\mathrm{SDD}_{\mathrm{SM}}$ es $\geq 2$, la discrepancia entre ambos somatotipos medios es estadísticamente significativa $(\mathrm{p}<0.05)$ y, por lo tanto, existirán diferencias entre ambos grupos. Las anteriores fórmulas están adscritas al manual de cineantropometría de la FEMEDE y fue el utilizado en este estudio.

\section{RESULTADOS}

\section{Identificación de los somatotipos según modalidad o sexo}

A continuación se advierte que la muestra se distribuye en 7 somatotipos fundamentales de 13 clasificaciones posibles según Heath-Carter (Carter, 2002). De ellos se destacan para los masculinos el somatotipo Endomesomorfo con 7 atletas de kayak para un $63.6 \%$ y con 9 atletas de canoa para un 90\%. En general dentro de esta clasificación se asentaron 16 atletas masculinos para $53.3 \%$ del total de la muestra. En la clasificación Meso-endomorfo se ubicó el mayor monto de atletas femeninos de kayak, con un total de 5 para 55.5\%.

Tabla 1. Clasificación del somatotipo según la modalidad o sexo en la población estudiada. Cuba 2006

\begin{tabular}{|c|c|c|c|c|c|c|c|c|}
\hline \multirow{3}{*}{ CLASIFICACION } & \multicolumn{4}{|c|}{ MASCULINO } & \multirow{2}{*}{\multicolumn{2}{|c|}{$\begin{array}{c}\text { FEMENINO } \\
\text { Kayak } \\
\end{array}$}} & \multirow{2}{*}{\multicolumn{2}{|c|}{ Total }} \\
\hline & \multicolumn{2}{|c|}{ Kayak } & \multicolumn{2}{|c|}{ Canoa } & & & & \\
\hline & No. & $\%$ & No. & $\%$ & No. & $\%$ & No. & $\%$ \\
\hline Endomorfo balanceado & - & - & - & - & 1 & 11.1 & 1 & 3.3 \\
\hline Endomorfo-ectomorfo & 1 & 9.1 & - & - & - & - & 1 & 3.3 \\
\hline Endo-mesomorfo & 7 & 63.6 & 9 & 90 & - & - & 16 & 53.3 \\
\hline Mesomorfo balanceado & 2 & 18.2 & - & - & - & - & 2 & 6.6 \\
\hline Mesomorfo-endomorfo & 1 & 9.1 & 1 & 10 & 2 & 22.2 & 4 & 13.3 \\
\hline Meso-endomorfo & - & - & - & - & 5 & 55.5 & 5 & 16.7 \\
\hline Ecto-endomorfo & - & - & - & - & 1 & 11.1 & 1 & 3.3 \\
\hline Total & 11 & 36.7 & 10 & 33.3 & 9 & 30 & 30 & 100 \\
\hline
\end{tabular}

\section{Análisis de los componentes del somatotipo medio según modalidad y sexo}

En el sexo femenino, se observa cómo los componentes derivan en un somatotipo Meso-Endomórfico (gráfico 1); es decir, que existe un predominio del sistema vegetativo y tendencia a la obesidad, aunque la masa muscular prevalece sobre las medidas longitudinales. El SDI $=4.14$ lo que se traduce en que no existe homogeneidad en (este párrafo hace alusión a los resultados con la muestra.) las féminas, por lo que existen diferencias significativas $(\mathrm{p}<0.05)$ entre los somatotipos de los individuos que integran la muestra.

En el sexo masculino, de la especialidad de kayak el somatotipo medio de estos atletas fue Endo-Mesomórfico. Se caracteriza por presentar un moderado desarrollo músculo-esquelético, pero con mayor peso específico y un nivel de grasa que predomina sobre la linealidad. Este grupo presenta un SDI=2.48 lo que significa que existen diferencias significativas $(\mathrm{p}<0.05)$ en cuanto a la homogeneidad de los individuos estudiados, aunque en menor grado que en las kayakistas. 
URL www.una.ac.cr/mhsalud

Gráfico 1. Somatotipo medio de la población estudiada según modalidad o sexo. Cuba 2006

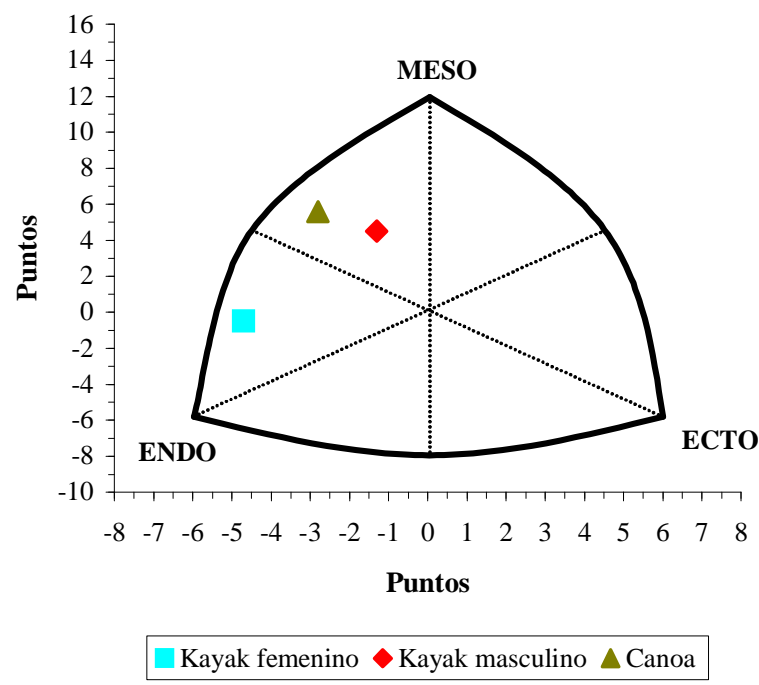

Entre las características distintivas del grupo de canoa, se determinó que tienen el mayor valor mesomórfico y el menor ectomórfico, presentando una moderada adiposidad relativa, con un alto desarrollo músculo esquelético, diámetros óseos y articulaciones grandes; es decir, gran volumen por unidad de altura, lo que determina un somatotipo Endo-Mesomórfico. Este grupo también presenta diferencias $(\mathrm{p}<0.05)$ en cuanto a la homogeneidad, pues exhiben un SDI=2.56, a pesar que diferentes estudios de investigación según plantea Berral (2005a), afirman y confirman la presencia de somatotipos semejantes para determinado deporte, así mismo, el somatotipo es más homogéneo en deportes individuales que en los de equipo, con la excepción del ciclismo y el tenis.

Entre grupos se observó que existe un dimorfismo sexual, dado porque las féminas muestran una superior adiposidad relativa entre 2-3 unidades y valores inferiores de mesomorfismo entre 1-2 unidades con respecto a los hombres. Al determinar $\mathrm{SDD}_{\mathrm{SM}}=7.41$ se aprecia una marcada dispersión entre ellos (Ver tabla 2).

Tabla 2. Distribución de los componentes del somatotipo medio, según la modalidad o sexo en la población estudiada. Cuba 2006

\begin{tabular}{lccccccc}
\hline \multirow{2}{*}{ Modalidad } & \multicolumn{2}{c}{ Endomorfia } & \multicolumn{2}{c}{ Mesomorfia } & \multicolumn{2}{c}{ Ectomorfia } & \multirow{2}{*}{ SDD $_{\text {SM }}$} \\
\cline { 2 - 7 } & $\mathbf{X}$ & SD & X & SD & X & SD & 7.41 \\
\hline Kayak mujeres & 6.8 & 1.49 & 4.2 & 0.88 & 2.1 & 1.17 & 2.82 \\
\hline Kayak hombres & 3.6 & 0.52 & 5.2 & 1.08 & 2.3 & 0.65 & 2.82 \\
\hline Canoa & 4.6 & 0.76 & 6.0 & 0.83 & 1.8 & 0.71 & 2.8 \\
\hline
\end{tabular}

Las dos especialidades del sexo masculino son similares en cuanto a somatotipo, evidenciándose que ambos tienen valores moderados de endomorfia. La valoración de la homogeneidad de estas dos disciplinas $\left(\mathrm{SDD}_{\mathrm{SM}}=2.82\right)$ muestra que los hombres son menos dispersos, aunque no homogéneos pues $\mathrm{SDD}_{\mathrm{SM}} \geq 2$; $\sin$ embargo, otros estudios plantean que el somatotipo es más homogéneo en deportes individuales que en los de equipo, con la excepción del ciclismo y el tenis (Garrido \& González, 2005). Por lo que se puede ultimar que en ambos sexos de esta selección no existe un modelo de somatotipo. Se puede observar que el valor más bajo de

Revista MHSalud® (ISSN: 1659-097X) Vol. 4. No. 2. Diciembre, 2007. 
URL www.una.ac.cr/mhsalud

ectomorfia lo tiene la canoa, por ello su característica distintiva en relación con las otras modalidades que aquí se describen.

\section{DISCUSIÓN}

\section{Correlación entre las variables resultado deportivo y componentes del somatotipo}

La intención en este acápite, es determinar la magnitud y dirección de la correlación existente entre los componentes del somatotipo y el resultado deportivo como variable dependiente (criterio de resultado deportivo en anexo 1). Es válido aclarar que este análisis se realizó considerando las distribuciones obtenidas en la totalidad de la población estudiada, en ambos sexos y en todas las modalidades.

Tabla 3. Coeficiente de correlación entre las variables resultado deportivo y componentes del somatotipo de la población estudiada. Cuba 2006

\begin{tabular}{cccc}
\hline $\begin{array}{c}\text { Coeficiente correlación Spearman } \\
(\mathrm{n}=30)\end{array}$ & Endomorfia & $\begin{array}{c}\text { Componentes del somatotipo } \\
\text { Mesomorfia }\end{array}$ & Ectomorfia \\
\hline Resultado deportivo & -0.179 & $0.413^{(*)}$ & 0.012 \\
\hline$* \mathrm{p}<0.05$ & & & \\
\hline
\end{tabular}

En la tabla 3, se observa que existe una correlación estadísticamente significativa, sólo entre el resultado deportivo y el componente mesomórfico. Ello se corrobora en el coeficiente de correlación obtenido, en el que se obtiene un valor de 0.413 , que resulta significativo con un nivel de confianza del $95 \%$. Si se parte de que este estudio se realiza a inicios del período preparatorio donde los atletas que integran la muestra solo acumulan pocas semanas de entrenamiento, se puede pronosticar que este valor irá en ascenso y la correlación será más significativa a medida que las cargas de entrenamiento se incrementen.

Estudios dedicados al análisis de datos antropométricos, citados por Berral (2005b), advierten que el componente muscular está relacionado con un mayor rendimiento deportivo y el endomórfico presenta una correlación negativa, demostrándose de igual forma en el estudio que sólo los atletas de canoa que son los que más altos valores de mesomorfia ostentan, han alcanzado ser medallistas en varias Copas del Mundo y Campeonatos Mundiales. Pudiendo explicarse además la razón por la cual las femeninas, con elevados valores de endomorfia, no han logrado insertarse dentro de las competiciones de élite mundial.

Otros estudios señalan que va a existir una ectomorfia mayor en deportes con grandes volúmenes de entrenamiento aeróbico (Esparza, 1993 y Garrido y González, 2005), pero recuérdese como afirman Labrada (2001) y Romero (2004), que se está en presencia de un deporte con una duración máxima de sus eventos de aproximadamente 4 minutos y que se desarrolla en un área funcional aeróbica-anaeróbica, donde los entrenamientos están dirigidos a aumentar la capacidad aeróbica, que servirá como base para el trabajo anaeróbico.

Por tales afirmaciones, se considera que prevalece el componente mesomórfico en este deporte, debido a las características metodológicas y a las exigencias energéticas del mismo, predominando así la intensidad de las cargas de entrenamiento en el proceso de planificación y dosificación. De aquí que el canotaje sea uno de los deportes con un elevado valor en el componente mesomórfico (Garrido \& González, 2005).

A tono con estos resultados, se puede concluir que, a mayores valores del componente mesomórfico corresponden resultados deportivos más relevantes, o lo que es lo mismo, existe una relación directamente proporcional entre ambas variables (gráfico 2). 
URL www.una.ac.cr/mhsalud

Gráfico 2. Correlación entre resultado deportivo y componente mesomórfico. Cuba 2006

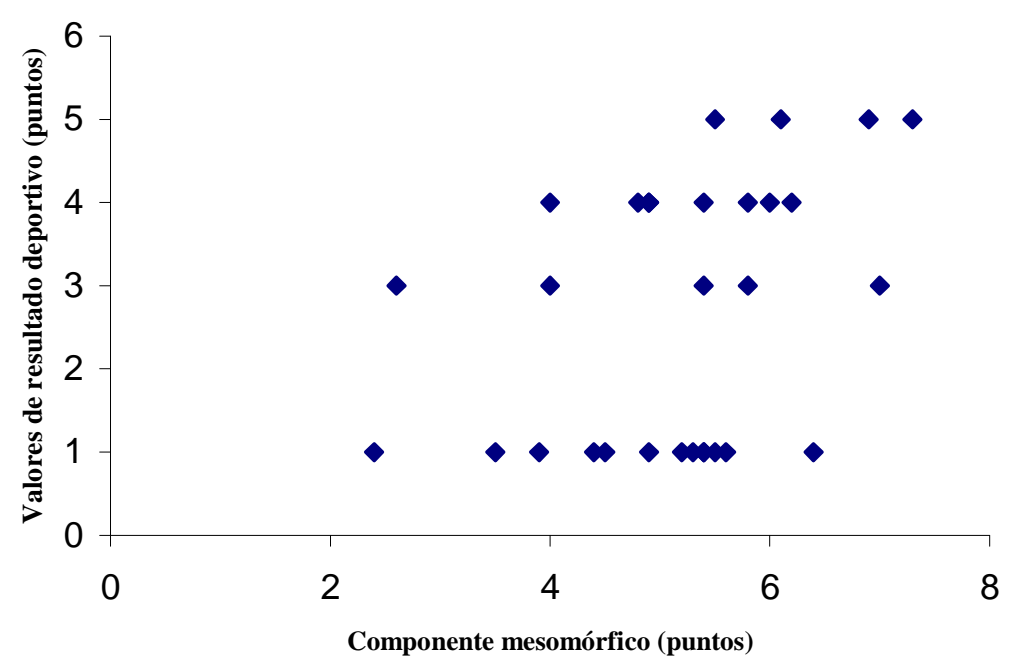

Análisis de los somatotipos medios de referencia internacional en el sexo femenino y su comparación con la muestra cubana

En el gráfico 3, se puede apreciar que en estudio realizado por Ackland y otros (2000) en los Juegos Olímpicos de Sydney, se analizaron 20 palistas femeninas, con un promedio de edad de 26.5 años, todas finalistas y/o medallistas en dicha competición, y se pudo comprobar que la clasificación media del somatotipo fue Mesomórfico Balanceado. Si se describen físicamente se puede decir que tienen poca grasa subcutánea, moderado desarrollo, músculo esquelético y linealidad relativa, a juzgar por la distribución de los componentes. Este gráfico fue confeccionado a partir de los datos que mostraban los estudios. La somatocarta hace una representación gráfica de la ubicación de los somatotipos y la tabla 4 muestra los valores de ellos. 
URL www.una.ac.cr/mhsalud

Gráfico 3. Comparación del somatotipo medio de Cuba con los de referencia internacional en el sexo femenino. Cuba 2006

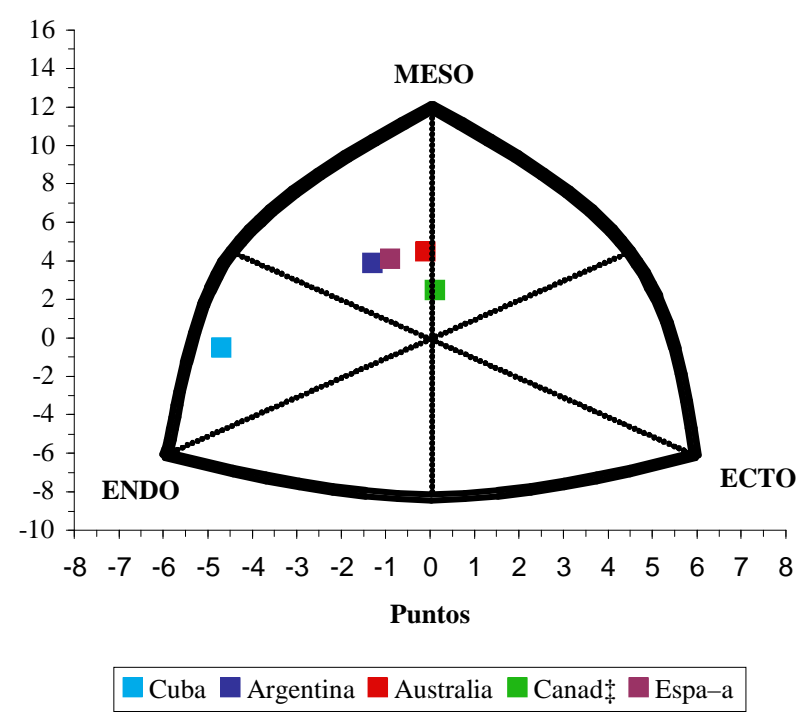

Fuente:

Australia (Ackland y otros 2000)

Canadá (Carter, 2002)

Argentina (Lentini y otros, 2006)

España (Esparza, 1993)

EE.UU. (Vaccaro y otros, 2000)

Esto mismo ocurre en la investigación de Montreal (Carter, 2002), donde fueron objeto de estudio 7 palistas competidoras, destacándose valores de la ectomorfia inferiores en 0.6 unidades, por ende estos atletas exhiben mayor volumen por unidad de altura.

Por otra parte, según una investigación realizada en el Centro Nacional de Alto Rendimiento Deportivo de Buenos Aires, Argentina (Lentini y otros, 2006), a 22 palistas femeninas con un promedio de edad de 18.5 años, se determinó un somatotipo Endo-Mesomórfico.

Tabla 4. Comparación de los componentes del somatotipo medio con la referencia internacional en el sexo femenino. Cuba 2006

\begin{tabular}{lccccccc}
\hline \multicolumn{1}{c}{ EQUIPOS } & \multicolumn{2}{c}{ ENDOMORFA } & \multicolumn{2}{c}{ MESOMORFIA } & \multicolumn{2}{c}{ ECTOMORFIA } & CLASIFICACIÓN \\
& $\mathbf{X}$ & SD & $\mathbf{x}$ & SD & $\mathbf{x}$ & SD & \\
\hline $\begin{array}{l}\text { Sidney (Ackland y otros } \\
\text { 2000) }\end{array}$ & 2.4 & 0.60 & 4.6 & 0.80 & 2.3 & 0.80 & Mesomórfico-balanceado \\
Montreal (Carter, 2002) & 2.8 & 0.26 & 4.1 & 0.78 & 2.9 & 0.58 & Mesomórfico-balanceado \\
Argentina (Lentini y otros, & 3.4 & 1.10 & 4.7 & 1.20 & 2.1 & 0.90 & Endo-mesomórfico \\
2006) & & & & & & & \\
España (Esparza, 1993) & 3.0 & 0.86 & 4.6 & 1.16 & 2.1 & 0.90 & Endo-mesomórfico \\
Cuba & 6.7 & 1.49 & 4.1 & 0.88 & 2.2 & 1.17 & Meso-endomórfico
\end{tabular}

En su apariencia física, en cuanto a las atletas argentinas se observa moderada adiposidad, la grasa subcutánea cubre los contornos musculares y óseos, existe un moderado desarrollo músculo esquelético, es decir, volumen muscular que predomina sobre las medidas longitudinales. Este equipo guarda estrecha relación con el de España, el cual fue estudiado por la Dra. Canda Moreno (Esparza, 1993) y como se puede observar el somatotipo 
URL www.una.ac.cr/mhsalud

medio de 6 palistas analizadas es Endo-Mesomórfico, pero la endomorfia muestra 0.4 unidades menos (ver tabla 4)

Se puede afirmar que existe una gran semejanza entre las medias de los equipos de referencia, predominando siempre el componente mesomórfico. Esta similitud se debe a que mientras mayor sea el nivel de los atletas más cerca se encontrará su ubicación en la somatocarta, o lo que es lo mismo existirá mayor homogeneidad entre los grupos (Esparza, 1993).

Al comparar los resultados de está investigación con los equipos de referencia, se observa una similitud de los componentes segundo y tercero del somatotipo, no siendo así en la endomorfia donde se encuentra una marcada diferencia alcanzando valores de más de 3 unidades. Obsérvese el evidente predominio del componente endomórfico (gráfico 3).

Si se analiza $\left(\operatorname{SDD}_{\mathrm{SM}} \geq 2\right)$ se puede percibir que existen discrepancias entre el equipo de Cuba y los de referencia (tabla 5).

Tabla 5. Distancia de dispersión de los somatotipos medios para el sexo femenino. Cuba 2006

\begin{tabular}{lccc}
\hline Equipos & $\begin{array}{c}\text { Australia } \\
\text { Ackland y otros (2000) }\end{array}$ & $\begin{array}{c}\text { Canadá } \\
\text { Carter (2002) }\end{array}$ & $\begin{array}{c}\text { Argentina } \\
\text { Lentini y otros (2006) }\end{array}$ \\
\hline Cuba & 9.41 & 8.84 & 9.44 \\
\hline
\end{tabular}

\section{Análisis de los somatotipos medios de referencia internacional en el sexo masculino y su comparación con la muestra cubana}

En estudio realizado en EE.UU. (Vaccaro, L y otros, 2000), a 13 atletas se pudo comprobar que la clasificación media del somatotipo fue Mesomórfico Balanceado. Si se describe físicamente se puede decir que tienen moderada grasa subcutánea, moderado desarrollo músculo esquelético y linealidad relativa, gran volumen por unidad de altura (tabla 6).

Este equipo guarda estrecha relación con el de España, el cual fue estudiado por la Dra. Canda Moreno (Esparza, 1993) y como se puede observar el somatotipo medio de 24 palistas analizados es Mesomórfico Balanceado, en ellos se destaca un alto desarrollo músculo esquelético relativo, diámetros óseos grandes, músculos de gran volumen, dado que la ectomorfia es inferior en 0.6 unidades estos atletas muestran mayor volumen por unidad de altura.

Por otra parte, según investigación realizada en el Centro Nacional de Alto Rendimiento Deportivo de Buenos Aires, Argentina (Lentini y otros, 2006), a 47 palistas masculinos con un promedio de edad de 18.7 años, se determinó un somatotipo Ecto-Mesomórfico.

Físicamente son individuos con moderado desarrollo músculo esquelético, menos volumen por unidad de altura donde los contornos musculares y óseos son visibles por su baja adiposidad relativa. Obsérvese que este equipo está conformado por atletas con edades muy jóvenes, lo que quizá no les ha permitido lograr valores más altos de mesomorfia, como los revelados en un estudio realizado en los Juegos Olímpicos de Sydney (Ackland y otros, 2000), donde se analizaron 50 palistas masculinos, con un promedio de edad de 26.5 años, todos finalistas y/o medallistas en dicha competición, y se pudo comprobar que la clasificación media del somatotipo fue EctoMesomórfico. 


\section{MHSalud}

URL www.una.ac.cr/mhsalud

Al analizar su físico son atletas con alto desarrollo músculo esquelético relativo (músculos de gran volumen), poca grasa subcutánea y linealidad relativa. Percátese que este estudio se realiza en fase competitiva donde se ha elevado la intensidad de los entrenamientos para adquirir la forma deportiva y por ello los atletas exhiben altos valores de mesomorfia y muy poca grasa subcutánea, muestra de que portan una óptima forma según el aspecto físico. Además según su promedio de edad son atletas que se han sometido durante años a trabajos hipertróficos en su preparación física. Estos dos aspectos pudieron marcar la diferencia de 0.9 unidades en el componente ectomórfico con relación al estudio anterior.

Somatotipo casi similar se indica en la investigación de Montreal (Carter, 2002), donde fueron objeto de estudio 12 palistas competidores, clasificados también como Ecto-Mesomórficos. En su aspecto físico se destaca linealidad relativa moderada; o sea, son atletas más esbeltos.

Tabla 6. Comparación de los componentes del somatotipo medio con la referencia internacional en el sexo masculino. Cuba 2006

\begin{tabular}{lccccccc}
\hline \multicolumn{1}{c}{ EQUIPOS } & \multicolumn{2}{c}{ ENDOMORFIA } & \multicolumn{2}{c}{ MESOMORFIA } & \multicolumn{2}{c}{ ECTOMORFIA } & CLASIFICACIÓN \\
& $\mathbf{X}$ & SD & $\mathbf{x}$ & SD & $\mathbf{x}$ & SD & \\
\hline Sidney (Ackland y otros 2000) & 1.6 & 0.50 & 5.7 & 0.80 & 2.2 & 0.70 & Ecto-mesomórfico \\
Montreal (Carter, 2002) & 1.5 & 0.45 & 5.2 & 0.83 & 3.1 & 0.95 & Ecto-mesomórfico \\
$\begin{array}{l}\text { Argentina (Lentini y otros, } \\
\text { 2006) }\end{array}$ & 2.1 & 0.60 & 4.8 & 1.30 & 2.7 & 1.00 & Ecto-mesomórfico \\
España (Esparza, 1993) & & & & & & & \\
EE.UU. (Vaccaro y otros, 2000) & 2.3 & 0.59 & 5.8 & 0.80 & 1.8 & 0.56 & Mesomórfico-balanceado \\
Cuba & 2.8 & 0.57 & 5.2 & 0.96 & 2.4 & 0.93 & Mesomórfico-balanceado \\
\hline
\end{tabular}

Fuente:

Australia (Ackland y otros 2000)

Canadá (Carter, 2002)

Argentina (Lentini y otros, 2006)

España (Esparza, 1993)

EE.UU. (Vaccaro y otros, 2000)

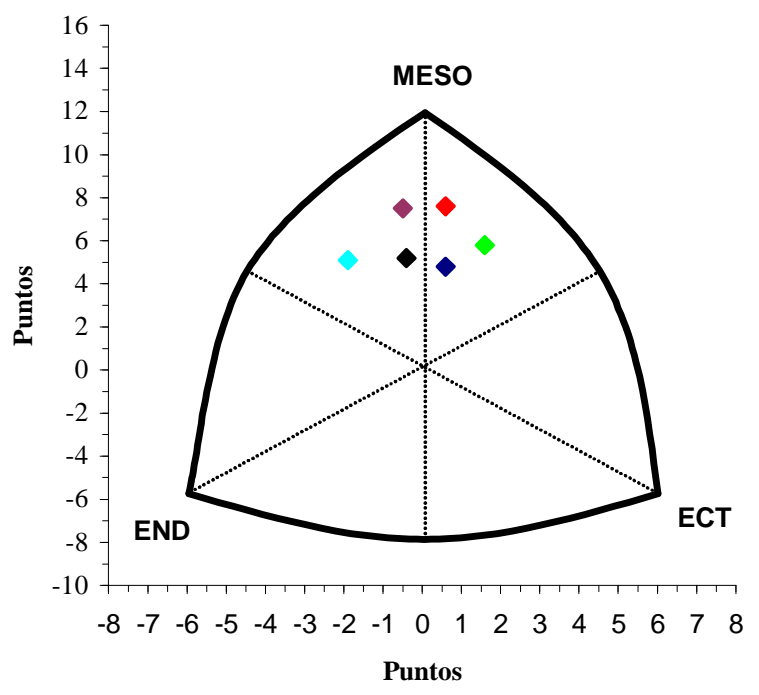

Argentina $\diamond$ Australia $\diamond$ Canadá $\diamond$ España $\diamond$ EE.UU. $\diamond$ Cuba 
URL www.una.ac.cr/mhsalud

Luego de realizar un análisis de los equipos de referencia se puede aseverar que existe similitud en cuanto al predominio del componente mesomórfico en todos los equipos de referencia, aunque en tres de estos también se destaca una moderada linealidad relativa.

Al confrontar los resultados del somatotipo medio masculino de este estudio con los equipos de referencia, se observa semejanza de los componentes segundo y tercero, no siendo así con la endomorfia donde se reveló una marcada diferencia, adquiriendo valores superiores a las 2 unidades (gráfico 4).

A juzgar por los valores de las distancias de dispersión de los somatotipos medios no existe homogeneidad dada por la diferencia $\left(\mathrm{SDD}_{\mathrm{SM}} \geq 2\right)$ entre la preselección de Cuba y los equipos tomados como referencia (tabla 7).

Tabla 7. Distancias de Dispersión de los Somatotipos medios para el sexo masculino. Cuba 2006

\begin{tabular}{lcccc}
\hline Equipos & $\begin{array}{c}\text { Australia } \\
\text { Acklandy otros } \\
(\mathbf{2 0 0 0})\end{array}$ & $\begin{array}{c}\text { Canadá } \\
\text { Carter (2002) }\end{array}$ & $\begin{array}{c}\text { Argentina } \\
\text { Lentini y otros (2006) }\end{array}$ & $\begin{array}{c}\text { España } \\
\text { Esparza (1993) }\end{array}$ \\
\hline Cuba & 5.00 & 6.10 & 4.34 & $\begin{array}{c}\text { EE.UU. } \\
\text { Vaccaro y otros } \\
(\mathbf{2 0 0 0})\end{array}$ \\
\hline
\end{tabular}

\section{Propuesta de acciones que contribuyan a modificar el somatotipo de la selección cubana de canotaje}

Conocer las limitaciones de ciertos físicos comparados con otros, permite establecer el punto de partida para la progresión de la instrucción física, así como las expectativas posibles de alcanzar un máximo rendimiento físico, basándose en los datos objetivos que aportan los estudios antropométricos.

Teniendo en cuenta que se está en presencia de un deporte donde el atleta necesita realizar un desplazamiento hidrodinámico de la embarcación y de su peso corporal, altos valores en el componente endomórfico disminuye los niveles de rendimiento (Sierra, 2003); por lo que se hace imprescindible proponer a los entrenadores una serie de acciones que modifiquen el componente endomórfico de estos atletas. Con el objetivo de acercar a los atletas cubanos al somatotipo que se describe en la literatura y los que exhiben equipos de élite mundial, se propone diseñar y ejecutar un programa para una pérdida segura de peso, donde en primera instancia se determinen las necesidades energéticas individuales que permitan establecer modificaciones en la dieta alimenticia, sin excluir el incremento de ejercicios aeróbicos como complemento dentro de la preparación física para lograr óptimos niveles del componente endomórfico.

Se considera que estos resultados no deben desestimarse a la hora de estructurar los programas de preparación de estos atletas en lo sucesivo, deben ser interpretados como fuente de retroalimentación continua, extendiéndose hasta lograr adecuados niveles de preparación en las distintas etapas del entrenamiento.

El somatotipo puede ser modificado en el orden individual y grupal gracias a acciones eficientes durante el entrenamiento deportivo, además orientan el principio de la diferenciación, que exhibe la teoría y metodología como campo científico del conocimiento. De aquí la esencia de la aplicación de este importante principio, que en apretada síntesis lo se reduciría a la siguiente expresión "a cada cual según lo que necesite”.

La aplicación práctica de este principio, se verá condicionada por el nivel de conocimiento que los entrenadores deportivos sean capaces de extrapolar a sus respectivos contextos. Para ello se hace necesario sistematizar este tipo de estudio desde una visión científica y bajo las condiciones que para ello se exigen, convirtiéndolos en focos de constante reflexión. 


\section{MHSalud}

URL www.una.ac.cr/mhsalud

Se trata pues, de hacer un uso racional de las aportaciones teóricas existentes en el campo de la cineantropometría, buscando una aproximación al "somatotipo ideal" o de referencia para el canotaje, aceptando que un piragüista presentará mayor rendimiento cuanto más semejante sea su descripción física a la del modelo de este deporte.

\section{CONCLUSIONES}

De la identificación de los somatotipos medios se obtuvo el Meso-Endomórfico para el kayak femenino, así como el Endo-Mesomórfico para el sexo masculino en las dos modalidades.

Las féminas mostraron una elevada adiposidad relativa con valores superiores a las 5.5 unidades y no son homogéneos sus somatotipos, puesto que $\mathrm{SDI}=4.14$. Los atletas masculinos tienen valores similares en sus componentes, predominando en ambos la endomorfia; de ellos los atletas de canoa ostentan mayor volumen por unidad de altura, con 0.8 unidades más en el componente mesomórfico y tan solo 1.8 unidades en el ectomórfico.

En ambas modalidades del sexo masculino los somatotipos son menos dispersos pero no homogéneos. El SDD $\mathrm{SM}_{\mathrm{SM}}$ mostró que los somatotipos de las féminas son más dispersos con respecto a los masculinos con un valor de 7.41; demostrándose así que en ambos sexos de esta selección no existe un modelo de somatotipo.

Únicamente se probó que existiera correlación entre el resultado deportivo de los atletas de la preselección cubana y el componente mesomórfico de los mismos, con un valor de 0.413 , que resulta significativo con un nivel de confianza del $95 \%$; aseverando, que un predomino de los componentes primero y tercero no se corresponden con mayores rendimientos en este deporte.

La comparación entre los somatotipos medios de referencias internacionales y los que se obtuvieron de la preselección cubana, muestra las diferencias que existen entre ambos, sobre todo en el componente endomórfico de este último, en el que se determinó 4 unidades más en el sexo femenino y 2.5 unidades en el sexo masculino, ratificando así, que estos no muestran valores óptimos en dicho componente lo cual los distancia del somatotipo ideal o de referencia para este deporte, aunque se comprobara la semejanza existente de los componentes segundo y tercero en ambos sexos.

Teniendo en cuenta lo perjudicial que representan altos valores en el componente endomórfico para este deporte, es necesario encauzar el entrenamiento sobre la base de incrementar los ejercicios aeróbicos y modificar la dieta alimenticia, con el fin de lograr una pérdida segura de peso en estos atletas y así crear una redistribución entre los componentes del somatotipo, lo cual mejoraría el rendimiento deportivo de los mismos.

\section{REFERENCIAS}

Ackland, T., Kerr, D., Hume, P., Norton, K., Ridge, B., Clark, S., Broad, E. \& Ross, W. (2000). Anthropometric normative data for Olympic rowers and paddlers. The Australian Journal of Science and Medicine in Sport, 1:22-24.

International Standards for Anthropometric Assessment Unerdale (2001). Manual Oficial de Cineantropometría ( $1^{a}$ ed.). Sydney, Australia.

Berral de la Rosa, J. (2005a). Somatotipo de los atletas. Extraído el 5 de Noviembre, 2006 del sitio Web de Universidad de Córdoba: http://webs.ono.com/usr000/nutridepor/pagina_nueva_36.htm.

Berral de la Rosa, J. (2005b). Protocolo de medidas antropométricas. Extraído el 5 de Noviembre, 2006 del sitio Web de Universidad de CÂّ3rdoba: http://webs.ono.com/usr000/nutridepor/pagina_nueva_37.htm. 


\section{MHSalud}

URL www.una.ac.cr/mhsalud

Carter, L. (1984). The somatotypes of athletes. A review. Human Biology: Cambridge 42:535-569.

Carter, L. (1990). Somatotype of paddlers competitors at Montreal. Extraído el 09 de diciembre 2006 del sitio Web: www.gssiweb-sp.com/sportssciencecenter.

Carter, L. (2002). The Heath-Carter anthropometric somatotype. Instruction manual [El somatotipo antropométrico de Heath-Carter. Manual de instrucción]. California: Editorial Universidad de San Diego.

Esparza, Ros F. (1993). Manual de cineantropometría. Pamplona: FEMEDE.

García, P. \& Rodríguez, A. (2004). Rendimiento Humano, Deporte y Salud: De la teoría a la práctica. Caracas: Ediciones Opsu.

Garrido, R y González, M. (2005). Correlación entre los componentes del somatotipo y la composición corporal según fórmulas antropométricas. Educación física y deportes. Año 10- No. 84. Extraído el 3 de Diciembre, 2006 de: http://www.efdeportes.com/

Gris, G. M. (2001). Componentes del somatotipo y ecuaciones antropométricas. Apunts Medicina de l'esport, 137:5-16.

Labrada, O. (2001). Evolución de la canoa en Cuba. Manuscrito no publicado.

Lentini, N., Gris, G., Cardey, M., Aquilino, G y Dolce, P. (2006) Estudio Somatotípico en Deportistas de Alto Rendimiento de Argentina. PubliCE Standard, Pid: 738. Extraído el 11 de Enero, 2007 de: http://www.sobreentrenamiento.com/PubliCE/.

Martínez, A. J. (1987). Antropología Física: el hombre y su medio. La Habana: Editorial Científico-Técnica.

Matveev, L. P. (2000). Teoría general del entrenamiento deportivo. Barcelona: Editorial Paidotribo.

Parajón, M. (2002). La Evaluación Antropométrica. PubliCE Standard. Extraído el 11 de Diciembre, 2006 de: http://www.sobreentrenamiento.com/PubliCE/.

Romero, E. (2001). Principios fundamentales para la selección y preparación atlética en deportistas de canoakayak. Manuscrito no publicado.

Romero, E. (2004). Características de cambios fisiológicos en las diferentes zonas de potencia en el desarrollo de las capacidades del rendimiento en el entrenamiento de canoa-kayak. Manuscrito no publicado.

Sierra, J. (2003). Elementos técnicos de la remada de kayak. Manuscrito no publicado.

Vaccaro, L., James, C., Richard, T. (2000). Somatotype of United States National Kayak (K2) Team. Extraído el 9 de Diciembre, 2006 de: http://www.medic.usm.my/.

Fecha de recepción del artículo: 04 de julio del 2007.

Fecha de aceptación del artículo: 5 de diciembre del 2007.

Fecha de publicación del artículo: 31 de diciembre del 2007.

Revista MHSalud® (ISSN: 1659-097X) Vol. 4. No. 2. Diciembre, 2007. 
URL www.una.ac.cr/mhsalud

\section{ANEXO 1}

\section{FICHA ANTROPOMÉTRICA}

\section{DATOS GENERALES:}

Nombre y Apellidos:

Edad:

Sexo:

$\mathrm{M} \_\mathrm{F}$

Longevidad deportiva (años):
Modalidad: __ Kayak __ Canoa

Tiempo en la selección (años):

Resultados deportivos relevantes:

1. Medallista de Oro en Campeonatos Nacionales y Olimpíada del Deporte Cubano.

2. Medallista de Oro en Juegos Deportivos del ALBA y Juegos Centroamericanos.

3. Medallista en Campeonatos Panamericanos del Deporte.

4. Medallista en Juegos Panamericanos y Pre-olímpicos.

5. Medallista o finalista en Copas del Mundo y Campeonatos Mundiales.

6. Medallista o finalista en Juegos Olímpicos.

\section{DATOS ESPECÍFICOS:}

Peso $(\mathrm{Kg})$ :

Talla $(\mathrm{cm})$ :

Pliegues (mm): - Tríceps:

- Subescapular:

- Suprailíaco:

- Pierna:

Perímetros $(\mathrm{cm}): \quad$ B Bazo contraído:

- Pierna:

Diámetros $(\mathrm{cm})$ : $\quad$ - Biepicondíleo de húmero:

- Bicondíleo de fémur:

Fecha: 
URL www.una.ac.cr/mhsalud

ANEXO 2

RELACIÓN DE ATLETAS CON RESULTADOS DEPORTIVOS RELEVANTES

\begin{tabular}{|c|c|c|}
\hline Nombre & Resultado & Competición \\
\hline \multirow{2}{*}{ Andy Sicilia } & Medalla de Oro, K-4 1000 mts. & Juegos Panamericanos de Santo \\
\hline & Medalla de Oro K-4 500 mts. & $\begin{array}{ll}\text { Domingo, } & \text { República } \\
\text { Dominicana. } 2003 & \end{array}$ \\
\hline \multirow{2}{*}{ Oslay Calzadilla } & Medalla de Oro, K-4 1000 mts. & Juegos Panamericanos de Santo \\
\hline & Medalla de Oro K-4 $500 \mathrm{mts}$ & $\begin{array}{ll}\text { Domingo, } & \text { República } \\
\text { Dominicana. } 2003 & \end{array}$ \\
\hline \multirow[t]{2}{*}{ Aldo Pruna } & $\begin{array}{l}\text { Medalla de Plata, C-2 } 500 \mathrm{mts} \text {. } \\
\text { Medalla de Bronce, C-2 } 1000\end{array}$ & $\begin{array}{l}\text { Campeonato Mundial Júnior de } \\
\text { Curitiba, Brasil. } 2001\end{array}$ \\
\hline & & Copa Mundial Senior de \\
\hline \multirow[t]{7}{*}{ Serguei Torres } & $\begin{array}{l}\text { Medalla de Plata, C-2 } 200 \mathrm{mts} \text {. } \\
\text { Medalla de Plata, C-2 } 500 \mathrm{mts} \text {. }\end{array}$ & $\begin{array}{l}\text { Poznan, Polonia. } 2006 \\
\text { Campeonato Mundial Júnior de }\end{array}$ \\
\hline & Medalla de Bronce, C-2 1000 & Tokio, Japón. 2003 \\
\hline & $\begin{array}{l}\text { mts } \\
\text { Medalla de Plata, C-2 } 200 \text { mts. }\end{array}$ & $\begin{array}{l}\text { Campeonato Mundial Senior de } \\
\text { Bathas, Kazajastán. } 2005\end{array}$ \\
\hline & Medalla de Bronce, C-2 500 & Copa Mundial Senior de \\
\hline & Medalla de Plata, C-2 1000 mts. & Copa Mundial Senior de \\
\hline & $\begin{array}{l}\text { Medalla de Bronce, C-2 } 500 \\
\text { mts. }\end{array}$ & Duisburg, Alemania. 2006 \\
\hline & $\begin{array}{l}\text { Medalla de Plata, C-2 } 1000 \mathrm{mts} \text {. } \\
\text { Medalla de Bronce, C-2 } 500 \\
\text { mts. }\end{array}$ & \\
\hline \multirow[t]{2}{*}{ Raidel Ramos } & 5ta. Posición en Hit Final, C-2 & Copa Mundial Senior \\
\hline & $\begin{array}{l}1000 \text { mts. } \\
7 \text { ma. Posición en Hit Final, C-2 } \\
500 \text { mts. }\end{array}$ & Poznan, Polonia. 2006 \\
\hline Frank Calerón & Medalla de Plata, C-1 500 mts. & Copa Mundial Junior de \\
\hline
\end{tabular}

